\title{
Exploiting Discreteness for Switching in Arrays of Nonlinear Waveguides
}

\author{
Ole Bang* and Peter D. Miller $\dagger$ \\ The Optical Sciences Centre, The Australian National University, Canberra ACT 0200, Australia
}

Received March 7, 1996; accepted April 22, 1996

\begin{abstract}
A new approach to multiport switching in arrays of nonlinear waveguides is proposed. While other schemes have relied on suppressing the inherent transverse discreteness, this approach takes advantage of this feature of the array. One of the effects of discreteness is to keep intense beams trapped in a single waveguide for the length of the array. Switching may be achieved by using a controlled perturbation to displace such a trapped beam in the transverse direction. This displacement is quantized to an integer number of waveguides, thus allowing unambiguous selection of the output channel.
\end{abstract}

\section{Introduction}

All-optical signal processing using integrated nonlinear waveguide optics has many desirable features. In particular, it is possible to fabricate components that are small and capable of high speed operation, only limited in principle by the "turn-off" time of the material nonlinearity [1]. One of the basic tasks of all-optical signal processing is switching, the ultimate goal being to achieve dynamic, fully controlled selection of one output channel among many. Here we consider the possibility of multiport switching in an array of identical regularly-spaced nonlinear waveguides, as depicted in Fig. 1.

Assume that the number of waveguides in the array, $N$, is large and that all exhibit weak cubic nonlinearity due to the Kerr effect. Further assume the distance between waveguides, $d$, to be large enough to allow the field in each waveguide to be treated almost as though in isolation $(d \gg \delta)$. By almost we mean that $d$ should still be sufficiently small to allow the evanescent field tails in neighboring waveguides to overlap just enough to create a small linear coupling (power leakage). Under these assumptions it can be shown that to lowest order the stationary envelope of the electric field in the $n$th waveguide is governed by the discrete nonlinear

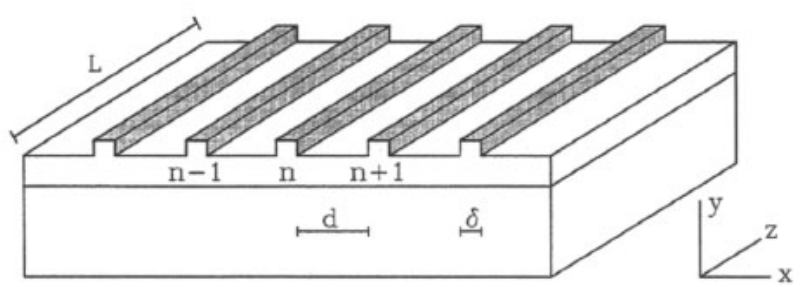

Fig. 1. Schematic diagram of part of an array of waveguides of length $L$. The width of each waveguide is $\delta$ and the spacing between waveguides is $d$.

\footnotetext{
* Email: oba124@rsphysse.anu.edu.au (O. Bang).

† Email: pdm124@rsphysse.anu.edu.au (P. D. Miller).
}

Schrödinger (DNLS) equation [2]

$\mathrm{i} \partial_{z} E_{n}+\left(E_{n+1}-2 E_{n}+E_{n-1}\right)+\left|E_{n}\right|^{2} E_{n}=0$,

here given in dimensionless units.

The N-core coupler is a device described by eq. (1) [2], in which switching is controlled by changing the power of a single-core input signal. Unfortunately, the power discrimination depends on the device length and decreases rapidly as the number of cores increases. In fact a complete power transfer into each of the output channels is no longer possible for more than three cores [3], and if the number of cores exceeds five, the ability to control the switching is lost [4]. A new approach is thus required if more than five output channels is desired. One alternative is to use the collective properties of the array and suppress its inherent discreteness by operating it with low intensity beams extending over several waveguides [4]. In this regime the array behaves essentially like a bulk medium and correspondingly beams are approximate spatial solitons of the continuous NLS equation. Thus a beam can propagate unhindered and emerge in a predictable region of the array, thereby selecting the output channel. In contrast to this approach, which we refer to as the continuum approach, we propose to exploit the discrete structure of the array by operating it at high intensities, where the effects of discreteness become especially apparent.

\section{Properties of a single beam}

The DNLS equation, eq. (1), has two conserved quantities, the total power and the Hamiltonian. Thus it is generally only completely integrable for $N \leqslant 2$, which explains the predictability of the two-core coupler [5]. For more than two cores the model may exhibit chaotic behavior, which e.g. leads to a high sensitivity of the three-core coupler to the device length [6]. However, even for large $N$, eq. (1) allows beams to exist that can propagate through the array in a regular and predictable way. These beams have properties that in several ways differ from those of beams propagating in a bulk medium.

Let us briefly outline the beam properties that are important for multiport switching in arrays. Without the nonlinear term, eq. (1) has linear plane-wave solutions of the form $\exp (i k n-i \beta z)$, where the propagation constant $\beta$ is related to the wavenumber $k$ by $\beta(k)=4 \sin ^{2}(k / 2)$. A packet of such plane-waves, with wavenumbers centered around $k$, will propagate in the array at an angle $\alpha(k)$, defined as $\tan (\alpha) \equiv \partial \beta / \partial k=2 \sin (k)$, and diffract subject to the linear diffraction coefficient $D(k) \equiv \frac{1}{2} \partial^{2} \beta / \partial k^{2}=\cos (k)$. At low 
maximum intensity $I_{\mathrm{m}}$ the primary combined effect of nonlinearity and positive diffraction is to allow for steady and uniform propagation of beams at the angle $\alpha(k)$. Neglecting terms of order $\mathcal{O}\left(I_{m}\right)$ the low intensity solution to eq. (1) can be found by multiple-scale techniques [7]

$$
\begin{aligned}
E_{n}(z) \simeq & \sqrt{I_{\mathrm{m}}} \operatorname{sech}\left(\sqrt{\frac{I_{\mathrm{m}}}{2 D}}[n-\tan (\alpha) z]\right) \\
& \times \exp \left(\mathrm{i}\left[k n-\left(\beta-\frac{1}{2} I_{\mathrm{m}}\right) z+\theta\right]\right) .
\end{aligned}
$$

The diffraction coefficient $D(k)$ does not appear if the second difference in eq. (1) is naively replaced by a second derivative, and indicates correctly how this approximate solution breaks down for wavenumbers above and close to the zerodiffraction wavenumber $|k|=\pi / 2$. Thus $\alpha(\pi / 2) \simeq 63.4^{\circ}$ represents an upper limit of the propagation angle of beams in the array, a fact that is not considered in the continuum approach. The constant phase $\theta$ will play the role of a switching parameter.

If the intensity is allowed to increase, the propagation of beams at angles $\alpha \neq 0$ will become impeded by collisions with the periodic transverse structure of the array. In fact, at sufficiently high intensities any beam will quickly become completely trapped and forced to propagate at an angle $\alpha=0$, regardless of its initial wavenumber. In this regime eq. (1) has exact stationary solutions that are remarkably stable and very localized. These solutions are fundamentally different from the solitary beams described by eq. (2) and play a key role in our approach to switching. No analytical formulas exist for such high intensity solutions. However, in the limit of large intensity, asymptotic methods can be applied to find the approximate solution $E_{n}(z)=\sqrt{I_{\mathrm{m}}} U_{n}$ $\exp (-\mathrm{i} \beta z)$, where $\beta$ and $U_{n}$ are given by

$\beta=2-I_{\mathrm{m}}\left[1+I_{\mathrm{m}}^{-2}+\mathcal{O}\left(I_{\mathrm{m}}^{-4}\right)\right]$,

$U_{\mathrm{n}}=\left\{\begin{array}{ll}1-\frac{1}{2} I_{\mathrm{m}}^{-2}+\mathcal{O}\left(I_{\mathrm{m}}^{-4}\right) & , \quad n=0 \\ I_{\mathrm{m}}^{-|n|}\left[1+\frac{1}{2} I_{\mathrm{m}}^{-2}+\mathcal{O}\left(I_{\mathrm{m}}^{-4}\right)\right], & n \neq 0\end{array}\right.$.

To improve this formula for finite values of $I_{\mathrm{m}}$ we use eq. (3) as an initial guess in a Newton-Raphson iteration scheme. This yields numerically exact solutions, trapped beams, in the whole intensity range of interest.

It is thus apparent that two distinct intensity regimes exist, in which beams can propagate through the array in a predictable way, and for switching applications it is important to identify them quantitatively. For arrays of length $L \leqslant 100$ the regimes

Angled beams : $I_{\mathrm{m}} \leqslant 0.2,|k|<\pi / 2$,

Trapped beams: $I_{\mathrm{m}} \geqslant 1.7$,

can be identified from numerical simulations of eq. (1). Here and in the following the number of waveguides $N$ is chosen to be large enough to avoid boundary effects.

\section{Controlled switching in the high intensity regime}

To exploit the effect of trapping for switching purposes the scheme must involve beams of maximum intensity higher than $I_{\mathrm{m}} \approx 1.7$. As pointed out above, such a trapped beam cannot move transversely in the array by itself. However, it may be displaced by a sufficiently large perturbation. Let us consider two such perturbations:
(A) Imposing a linear phase gradient at the input. Here the initial beam profile is a numerically exact solution close to eq. (3) with maximum intensity $I_{\mathrm{m}}^{\mathrm{hi}}$, which is then multiplied by exp (ikn) to initiate transverse propagation.

(B) Colliding with a low intensity angled beam given by eq. (2) with parameters $\left(I_{\mathrm{m}}^{\mathrm{lo}}, k, \theta\right)$, launched $\Delta n$ waveguides away from the trapped beam of maximum intensity $I_{\mathrm{m}}^{\mathrm{ht}}$.

Figure 2 shows representative examples of these two possibilities. In both cases the perturbation displaces the trapped beam in the transverse direction, but only an integer number of waveguides, due to its high intensity and the subsequent strong trapping imposed by the discrete structure of the array. We propose to take advantage of this transversely quantized displacement in switching applications.

Let us look closer at the switching process and how it can be controlled by making many simulations of the type presented in Fig. 2. In each simulation the trapped beam is incident on the array at $n=0$ and has the power $P_{\text {in }} \equiv \sum_{-3}^{3}\left|E_{n}(0)\right|^{2}$. At the output $z=L$ we measure the position $n_{\mathrm{c}}$ as the waveguide of maximum intensity, and define the contrast $C \equiv\left|E_{n_{\mathrm{c}}}(L)\right|^{2} /\left(\left|E_{n_{\mathrm{c}}-1}(L)\right|^{2}+\left|E_{n_{\mathrm{c}}}(L)\right|^{2}\right.$ $\left.+\left|E_{n_{\mathrm{c}}+1}(L)\right|^{2}\right)$ and the power loss $\Delta P \equiv\left(P_{\text {in }}-P_{\text {out }}\right) / P_{\text {in }}$, where $P_{\text {out }} \equiv \sum_{n_{\mathrm{c}}-3}^{n_{\mathrm{c}}+3}\left|E_{n}(L)\right|^{2}$.

Method A includes two control parameters, the maximum intensity $I_{\mathrm{m}}^{\mathrm{hi}}$ and the wavenumber $k$. The length of the array and the number of waveguides in the array are fixed design parameters. Figure 3 shows the displacement of the trapped beam as function of $I_{\mathrm{m}}^{\mathrm{bi}}$, with $k$ as a parameter. Clearly, the array can be operated as a power-controlled switch by using the plateaus on the displacement curves. Thus switching among up to 7 waveguides can be achieved for $k=-0.5$.

Generally the displacement depends on the strength of the perturbation compared to the strength of the trapping exerted on the beam by the discrete structure of the array. Thus the higher the value of $|k|$ and the lower the value of $I_{\mathrm{m}}^{\mathrm{hi}}$, the more the beam is displaced and the more sensitive the switching is to the control parameters. The contrast of the output beam lies between 0.43 and 0.82 , and the power loss is positive, varying between $3 \%$ and $11 \%$. Note that in all simulations, including the one in Fig. 2A, most of the loss

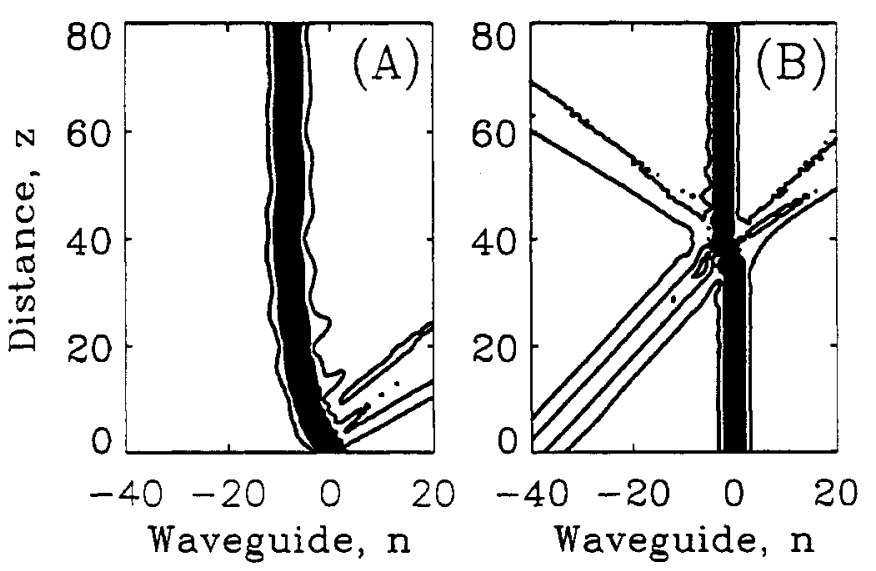

Fig. 2. Examples of methods A and B to displace a trapped beam. Each plot shows the contour of the intensity $\left|E_{n}(z)\right|^{2}$ found by numerical integration of eq. (1) with initial condition as explained in the text. Common parameters: $L=80, N=101$ and $I_{\mathrm{m}}^{\mathrm{bi}}=2.0$. Individual parameters: (A) $k=-0.52$ and (B) $I_{\mathrm{m}}^{\mathrm{lo}}=0.2, k=0.52, \theta=0$ and $\Delta n=40$. The displacement is (A) 8 waveguides and (B) 2 waveguides. 


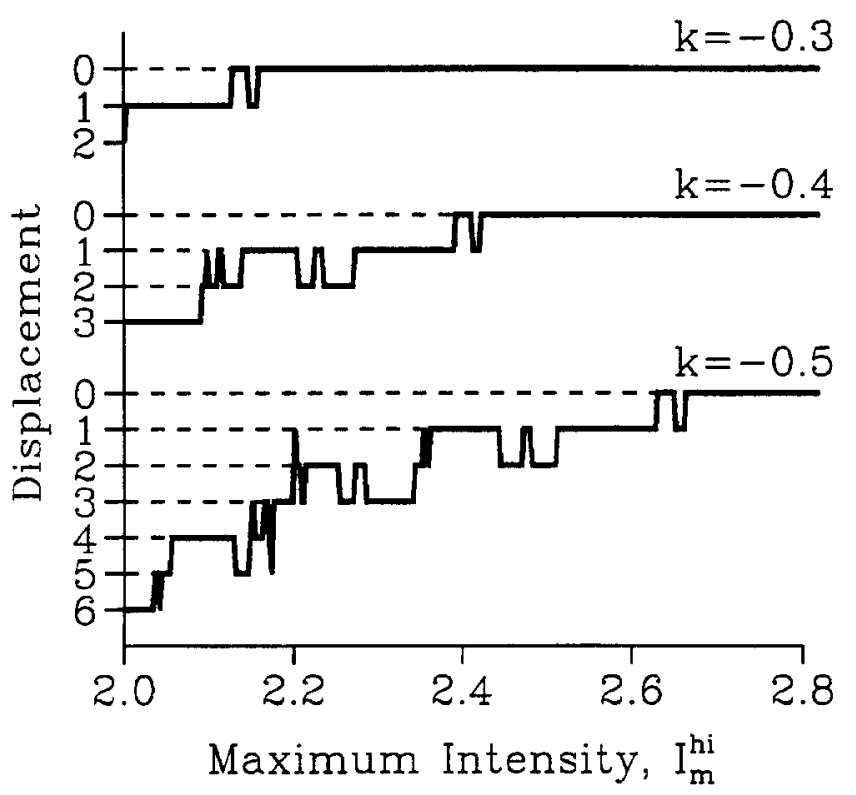

Fig. 3. Displacement of a trapped beam of maximum intensity $I_{\mathrm{m}}^{\mathrm{hi}}$ after being given a linear phase gradient $\exp (\mathrm{ikn})$ at the input, as function of $I_{\mathrm{m}}^{\mathrm{hi}}$ for different values of $k$. Results of numerical simulation of eq. (1) with initial condition as explained in the text. Fixed parameters: $L=40$, $N=101$.
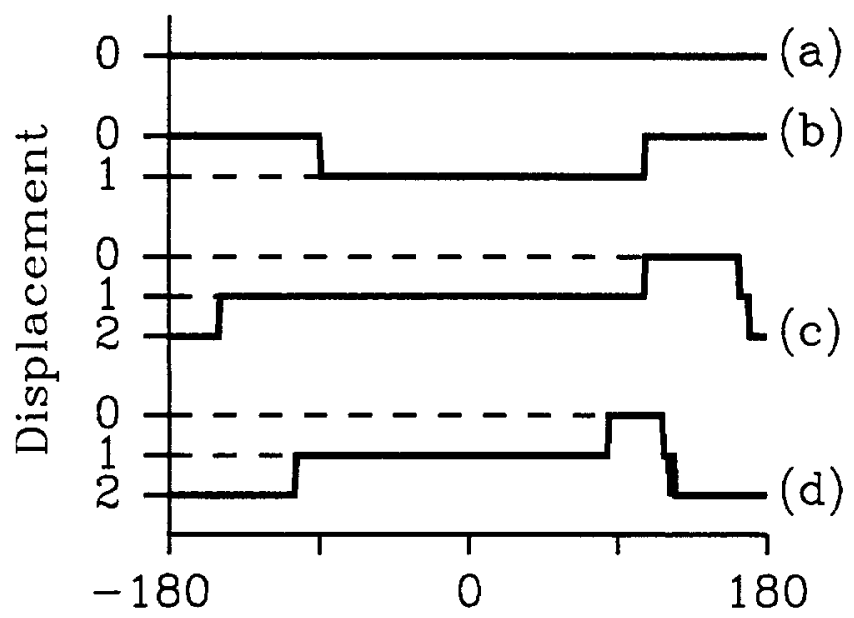

Phase constant, $\theta$

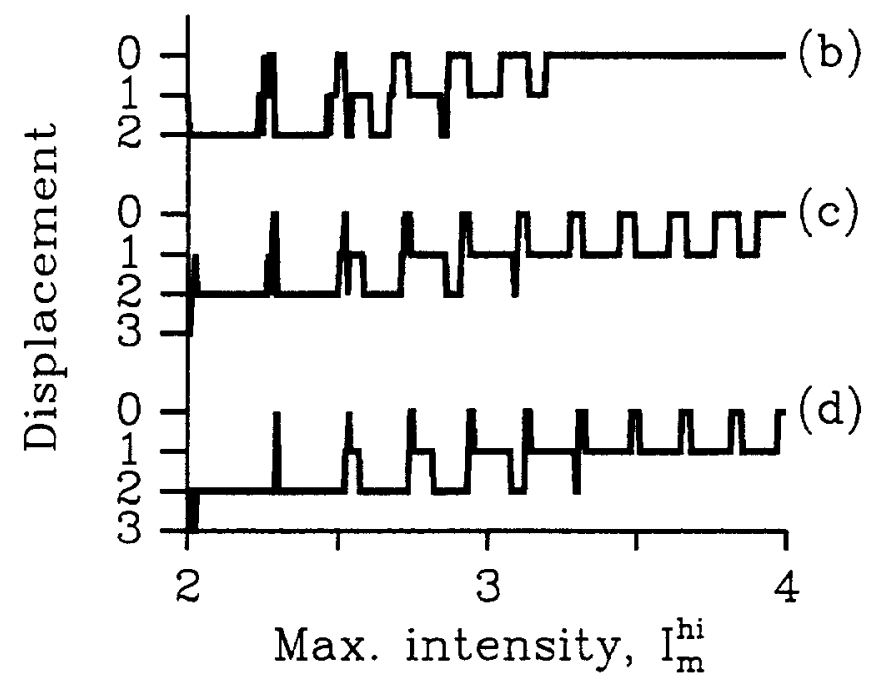

Fig. 4. The number of waveguides a trapped beam of maximum intensity $I_{\mathrm{m}}^{\mathrm{h}}$ is displaced after collision with an angled beam of maximum intensity $I_{\mathrm{m}}^{\mathrm{m}}=0.05$ (a), 0.1 (b), 0.15 (c) and 0.2 (d), as function of (top) the phase constant $\theta$ and (bottom) $I_{\mathrm{m}}^{\mathrm{ht}}$. Results of numerical simulation of eq. (1) with initial condition as explained in the text. Fixed parameters: $L=80$, $N=101, \Delta n=40, k=0.52$, and (top) $I_{\mathrm{m}}^{\text {th }}=3$ and (bottom) $\theta=0$. in power is converted into a low intensity beam that propagates through the array at an angle that increases with $|k|$.

Due to the presence of the colliding beam, method B has four control parameters, the maximum intensities $I_{\mathrm{m}}^{\mathrm{hi}}$ and $I_{\mathrm{m}}^{\mathrm{lo}}$, the wavenumber $k$ and the phase $\theta$. The array must be longer than with method $A$ because the center of action of the perturbation is no longer at the input, but at some distance determined by $k$ and the initial separation $\Delta n$ (see Fig. 2B). Thus we consider $L, N, k$ and $\Delta n$ all as fixed design parameters.

The top set of curves in Fig. 4 shows the displacement of the trapped beam as function of $\theta$ for different values of $I_{\mathrm{m}}^{\mathrm{lo}}$. Due to the relatively high intensity of the trapped beam, $I_{\mathrm{m}}^{\mathrm{hi}}=3$, switching can only be achieved among three waveguides, but the displacement curves are "cleaner" than in Fig. 3. Furthermore, the contrast is extremely good, lying between 0.74 and 0.89 . The power loss is now generally negative, $-10 \% \leqslant \Delta P \leqslant 0 \%$, and thus the input beam is amplifed by the collision process. This amplification seems to be a general mechanism in discrete systems [8]. The bottom set of curves in Fig. 4 shows how power-controlled switching can be achieved with method B. The oscillatory behavior is due to resonances between the intensitydependent propagation constants of the two beams. Contrasts range between 0.46 and 0.91 , and the input beam is still amplified by the collision, $-16 \% \leqslant \Delta P \leqslant 0 \%$.

\section{Discussion}

Controlled switching can thus be achieved in arrays of nonlinear waveguides operated in the high intensity regime, where the properties of beams differ drastically from those of beams in bulk media. Correspondingly, the approaches to switching presented here are fundamentally different from the continuum approach [4].

In the continuum approach the displacement of the input beam happens gradually over the whole length of the array. Thus the efficiency of the switching depends critically on the control of the angle, in that even slight variations change where the output signal is detected. The input contrast is $C=0.45$ for the maximum intensity $I_{\mathrm{m}}=1.1$ used in [4]. The output contrast will be somewhat less, in part because the maximum intensity is outside the regime, defined by eq. (4), for solitary beams.

By contrast, in both methods A and B the displacement of the trapped beam takes place in a small region in $(n, z)$ space, which means that the efficiency of the switch can be insensitive to variations of the array length. The input beam is extremely localized, and this sharp contrast is maintained at the output, $0.43 \leqslant C \leqslant 0.91$. As has been noted [9], maximizing the contrast is important for practical implementations. In addition, the two-beam method $B$ provides the constant relative phase $\theta$ as a novel control parameter.

\section{Acknowledgements}

This work has been supported by the Australian Photonics Cooperative Research Centre. Parts of this work have been published in Optics Letters [10].

\section{References}

1. Stegeman, G. I., "Nonlinear Waves in Solid State Physics" (Edited by A. D. Boardman) (Plenum Press, New York 1990), p. 463. 
2. Christodoulides, D. N. and Joseph, R. I., Opt. Lett. 13, 794 (1988).

3. Schmidt-Hattenberger, C., Trutschel, U. and Lederer, F., Opt. Lett. 16, 294 (1991).

4. Kròlikowski, W., Trutschel, U., Cronin-Golomb, M. and SchmidtHattenberger, C., Opt. Lett. 19, 320 (1994).

5. Jensen, S. M, IEEE J. Quantum Electron. QE-18, 1580 (1982).
6. Finlayson, N. and Stegeman, G. I., Appl. Phys. Lett. 56, 2276 (1990).

7. Bang, O. and Peyrard, M., Physica D 81, 9 (1995).

8. Bang, O. and Peyrard, M., Phys. Rev. E53, 4143 (1995).

9. Matsumoto, M., Katayama, S. and Hasegawa, A., Opt. Lett. 20, 1758 (1995).

10. Bang, O. and Miller, P. D., Opt. Lett. 21, 1132 (1996). 